\title{
THE ROLE OF REINSURANCE IN EARTHQUAKE RISK ASSESSMENT
}

\author{
B. Porro
}

\begin{abstract}
SUMMARY
This paper deals with the assessment of catastrophe perils from a reinsurance point of view. Taking earthquake as an example, we try to answer two crucial questions

- What are the factors that most influence the premium?

- How can we arrive at (maximum) event losses?

In a first paragraph, we try to define catastrophe losses in contrast to single risk losses. Then we discuss in some detail the elements that have the largest impact on catastrophe losses: size and frequency of earthquake events, vulnerability of the insured interests exposed and the conditions of the earthquake endorsement.

New Zealand is taken as an example to show how risk premiums can be calculated and how we can arrive at maximum probable/possible losses with a certain occurrence probability.

Finally, based on the questions we want to answer, the basic information needs in the entrepreneurial reinsurance environment are touched upon. The paper is intended as an introduction to the verbal presentation and the following discussion.
\end{abstract}

\section{INTRODUCTION}

If we look at lines such as fire, motor or engineering, there is a lot of actuarial literature about Insurance and Reinsurance. And always we find the more or less valid assumption of the risks being independent from each other. As soon as we consider natural perils, the assumption of mutually independent risks is no longer valid. This is the basic problem of what we call catastrophe perils: exposed sums of individual risks (fire and other lines) have to be added up, and if the geographical concentration of insured risks is high, we soon are confronted with breathtaking amounts of exposure. Another factor we are not used to in day to day business is the absence of regular losses. Many years can pass by during which premiums are collected without paying any claims. This makes it impossible to calculate premiums based on experienced losses. The upper limit of an event loss, theoretically the sum of all sums insured, plus a loading for (value added) taxes and

* Swiss Reinsurance Company Zurich, Switzerland loss adjustment costs, is considered unrealistic or impossible to happen. So we use EML factors to estimate our monetary commitment, without knowing what we actually do. In addition we can experience serious interline accumulations, also a situation that we are not used to in "normal" underwriting.

Is there any difference between an insurance and a reinsurance company with regard to catastrophe perils? Looking at the recent past, reinsurers have paid the overwhelming part of the original gross loss, over $90 \%$ in the Mexico and san Salvador events. If the company has been operating worldwide, reinsurers have at least had the opportunity to learn much more due to the fact that they have been hit in several instances over the last two decades, starting with Managua in 1972 and ending with the Edgecumbe event in 1987. Less than 20 years and an even smaller number of loss events are not much to develop and calibrate models. We have to admit that we still are on the top part of the learning curve, and therefore have to revise our findings by critical reconsideration of the facts after each event. The following lines therefore are a state of the art report and not a cooking recipe for underwriting catastrophe perils! 
Another difference between local insurer and international reinsurer may be the way they look at damage potential. For the insurer the underwriting concentrates on the individual risk, whereas the reinsurer - due to lack of enough detailed information - has to focus on whole portfolios. Only in very few instances it so far was possible to undertake a joint (insurer plus reinsurer) loss analysis of an earthquake occurrence. As a consequence the link from the individual claim to the gross loss and to the reinsured or retained loss amount is neither well known nor thoroughly understood.

\section{MAIN DIFFERENCES BETWEEN SINGLE} RISK LOSSES AND CATASTROPHE LOSSES

Just to make sure we speak the same language, let us describe the main characteristics of the two loss types:

- A single risk loss or individual loss limited to what an underwriter considers the maximum sum insured to be affected by one insured peril in one occurrence. Due to this subjective element, in some instances two underwriters will arrive at different risk definitions. To give an example: what constitutes one single risk in property insurance? One underwriter will consider all items insured with his company and situated within the same building or complex. The more conservative underwriter will check on possible conflagration with neighbouring items and also take the consequences of an explosion into account.
- Catastrophe losses are therefore either losses caused by an insured peril affecting more than one risk that was considered independent of all others in the underwriting (conflagration), or - what we will concentrate on - losses caused by perils other than the one used to define the single risk and by it's nature affecting more than one risk in one occurrence. In property insurance, all natural perils like wind, water (hail, snow, ice) and earthquake are well known examples of perils causing catastrophe losses.

To be more explicit, let's concentrate on fire and earthquake for analysing the main differences between single and catastrophe losses (see comparisons in Table 1).

Earthquake risk assessment therefore has to tackle technical as well as entrepreneurial problems, best expressed in the following questions we will try to answer provisionally:

- How should we calculate the risk premium?

- How large could an event loss become, and what is the corresponding ruin probability?

- What kind of information do we need for underwriting proportional and nonproportional treaties?

- How do cover terms and conditions influence the individual and total gross loss?

\section{Fire}

size of risk

Frequency of events

Area affected

Underwriting

know-how

Loss adjustment

and claims

management

Consequences one building or block

low for one risk, relatively high for large portfolio

less than $1 \mathrm{~km}^{2}$

damage factors known and reasonably well quantified

highly developed and specialised, many claims at any moment in time pending

Experience based rating possible

risk of conflagration very low

damage fluctuation decreases with increasing number of risks in portfolio

\section{Earthquake}

part of or whole portfolio

low for whole portfolio

1000 to $100.000 \mathrm{~km}^{2}$

generally poor due to lack of experience

improvisation, lack of specialists. Many claims at once, but very few events in an underwriter's lifetime

rating has to be based on semiempirical models

by definition high conflagration

damage fluctuation increases with growing portfolio size in given geographical area 
THREE FUNDAMENTAL ELEMENTS IN EARTHQUAKE RISK ASSESSMENT

We will discuss them individually, always looking from the insurance point of view.

\section{Seismology}

We are interested to know how many earthquakes are going to happen in the average in a specific area and how strong they will be. I would like to emphasize the words in the average, thereby denying all efforts to predict time, strength and place of an earthquake. If earthquake predictions were to become reliable, we should have to refuse the earthquake peril as an insurable line of business!

Earthquake reporting history can be divided into three distinct periods.

- First, the time before the turn of this century. Earthquake reports up to about that date consist of descriptions of effects to man, buildings and the environment. For better and standardised reporting, several intensity scales were introduced as from about 1850 (Rossi Forel 1885, Mercalli 1902). This period can be characterised as follows: only larger earthquakes reported, very strong subjective element in interpretation, lack of continuity (wars, migration, fires in archives), strong influence of myths (acts of God). In a nutshell: poor quality of reported information. To be used only after reassessment by experts.

- Second, the time span up to 1964. Gradual introduction of recording instruments of different type and sensitivity. Infancy of seismology with all problems inherent to new developments: rapid changes of instrument types, many of them maintained by interested. laymen, large differences from one country to the other as far as density of instruments and reporting are concerned. No standardized world time, therefore large errors in locating epicentres when using information from various observatories. Completeness of recording for small to medium sized magnitudes and for some areas gradual but slowly improving over considered time period.

- Third, introduction of the Worldwide Standardized Seismograph Network (WWSSN) in 1964. Complete registration on continents down to Magnitude 4.5 events. on the other hand partial disappearance of intensity assessment of area affected by trained seismologists.

In conclusion, seismology still is in an evolutionary phase, and we will have to wait some more years until information is collected and disseminated in a standardized format worldwide. For the time being, we have to depend on regional or even national catalogues, difficult to obtain, even more difficult to interpret as far as reassessment of preinstrumental history is concerned.

What information do we need as insurers in our risk assessment? Ideally, we would like to have for each event (at least for those able to cause insured damage) time and place (including depth) where it happened, amount of energy released during the rupture process (Magnitude) and the intensity distribution (using Modified Mercalli scale). With this information at hand, it is possible to estimate the direction of maximum and minimum intensity attenuation, the areas affected by various intensity levels for an earthquake of a given magnitude and depth. But we still depend on a very short instrumental observation period to calculate occurrencefrequencies, especially for medium to large magnitude earthquakes. Large uncertainties have to be expected in areas of low seismic activity, where return periods of several centuries are common for events with a high damage potential (ask an actuary how reliable a return period estimation is if you have one event within the 25 year observation period).

One question still remains open: Do earthquakes happen at more or less regular intervals (average constant over time), are there cycles (seismic gaps but still constant rate of occurrence over large periods) or is there a trend toward higher or lower seismicity?

For our example, we will use the "Revised Estimates of Earthquake Hazard in New Zealand" published in the Bulletin of the NZNSEE in December 1983. For the cities listed below, we get the following return periods:

MM VI MM VII MM VIII MM IX

$\begin{array}{lrrrr}\text { Auckland } & 62 & 260 & 1400 & \\ \text { Wellington } & 6 & 21 & 67 & 220 \\ \text { Nelson } & 5 & 16 & 56 & 200 \\ \text { Christchurch } & 14 & 48 & 160 & 600\end{array}$

\section{Vulnerability}

The crucial question is: Given an earthquake intensity (either MM-Intensity or a Magnitude indication plus distance and attenuation characteristics), what kind of damage do we have to expect as a function of building type and quality? The same question has to be answered for contents and Business Interruption covers.

only practical evidence can help us finding the answer, and by this we mean in-depth analysis of building stock and its affected part after a damaging event. Let's have a look at some hurdles we have to overcome in this process.

- Does anybody know an insurance company able to provide you with information on type of building, age, size, building quality, level of maintainance, materials used in construction, subsoil, foundation and geographical location?

- In general, earthquake cover is granted with an endorsement to the fire policy, and therefore building classification matches with the fire underwriting information and does not consider earthquake-shock relevent items. As an example, wooden buildings are heavy fire 
risks, but quite resistant to earthquake shock. The other extreme are brick houses without any consideration of seismic forces in the design but classified as good fire risks. Antiselection either in the retained part or in the ceded part of the risk is the consequence. If a portfolio is composed just of one building class, a substantial difference between the loss ratios of retained and ceded business can result, especially if the earthquake premium is expressed as a percentage of the fire premium or if a flat rate is charged independent of vulnerability.

- The loss amount is defined as being equal to the indemnification to policy holder plus (value added) taxes plus loss adjustment expenses. The indemnification depends very much on the insurance cover conditions (coinsurance; deductible; loss limit; items like debris removal, loss of rent etc. included or not). For a transparent and comparable loss analysis, the basis we would like to work with is:

Total cost of repair (one claim per building)

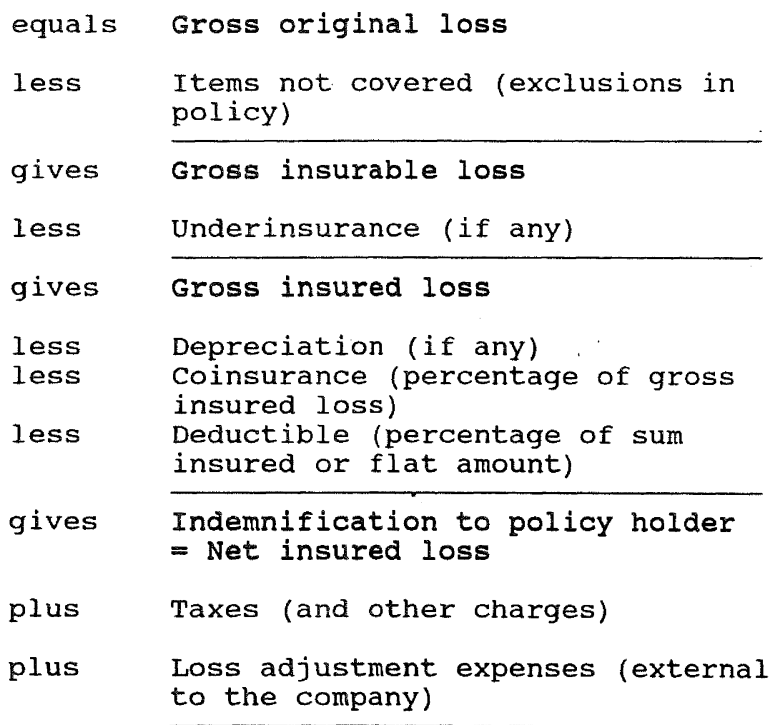

gives Total insured loss cost

Even with this ideal breakdown, differences from one country to another can be substantial. only careful consideration of all factors will show whether loss data can be transferred from one country to another without adjustments.

- Loss data investigations only make sense if losses are put into relation with the corresponding sums insured exposed in the event, and if broken down by building class and areas of comparable intensity. only then can a Mean Damage Ratio

$(M D R)=$ sums of all gross insured losses sum of all sums insured

be calculated that can be used in earthquake risk assessment. Items like taxes and loss adjustment expenses have to be considered separately, because they can vary substantially from one company and/or country to another.

- A very important factor in the calculation of MDR's is, whether (post earthquake) inflation is carried by the policy holder or the insurer. In some countries prices are frozen at date of the loss, whereas in others the full inflation impact has to be born by the insurance carrier. The resulting MDR's (per building class, per intensity degree) are average values! They only can be used to calculate expected losses, if the number of corresponding buildings in the portfolio is large. What does large mean? Without further qualifications, their number should exceed 100.

Some further remarks related to the classification of buildings with regard to vulnerability:

- building codes. In general terms, the improvement of building quality is a function of building codes' requirements. But for many reasons, the quality of codes is not reflected to the generally assumed level in the MDR's. Why? First, building codes almost exclusively deal with the load bearing structure, not with the nonstructural items. Second, the final intention of codes is not to reduce material damage, but to save lives by preventing the structure to collapse. Third, building codes are regulations that have to be followed not only on the construction plans to get the construction permit, but also during the erection of the structure. In many countries, the supervision and control is poor, with a corresponding decline in the quality of materials used and workmanship. Four, after an event codes tend to be revised upwards. This means, that the actual building code is only incorporated in structures completed about three to four years after the last earthquake hit the area. Five, structural losses cannot exceed 30 to 40 percent of the building value, for the nonstructural components account for the balance. Experience gained so far shows that structural losses in an area affected with intensities up to about MM VIII never exceeded 10 to $15 \%$, including structures built to old standards.

- Age is an important factor but very difficult to quantify, for with progressing time building structures have changed, other materials have been used, and living standards have gone up (reflected in non-structural items like heating, air conditioning, wiring). Investigations (Intensities up to MM VIII) have shown, that for comparable buildings age is a decisive factor and that MDR's of new buildings easily can be doubled to be representative for old ones. There is one exception: if the area has been hit by other events, only good quality buildings have survived and show a vulnerability similar to new structures. Maintenance can be considered as "face-lifting"; buildings look younger and behave better during earthquake shaking. 
- Subsoil. The influence of the subsoil on MDR's is not totally separable from building quality, design and dimensions. It is also dependent from building practice in a country, mainly on type of foundation used and existence of basements. For dwelling risks, it can be assumed that the basement compensates for less than average subsoil conditions, because resonance has not the impact as with multistory structures. If no basement exists, the anchoring of the superstructure to the foundation is the critical damage factor, because falling off the foundation piles usually leads to high losses.

Good foundations are required to prevent differential settlement on unconsolidated soils found near rivers or lakeshores and at the bottom of hills. Vulnerability increases for buildings located on hillsides, not only due to subsoil conditions and increased probability of earthslides but also for amplification of arriving earthquake waves and irregular shape of buildings on slopes.

- Height. In many tariffs, there is a loading for buildings depending on number of stories or height. If this loading is charged to compensate for the increased fluctuation of results (for the large eggs in a basket) that's fine. No empirical evidence is available to support this loading from a pure vulnerability standpoint except in the case of high buildings on deep alluvial soils like Lago di Texcoco in Mexico City or Los Palos Grandes in Caracas. In these instances, resonance effects can lead to large or even total losses as evidenced in the 1985 Mexico and the 1967 Caracas events.

- Regularity. Experience has shown that buildings of otherwise comparable quality become more vulnerable, the more they are irregular. By this we understand unsymmetrical ground plans, variations in floor area with height (set backs), different height of stories. Other irregularities are less visible, like irregular stiffness distribution leading to torsional oscillations during the earthquake motion, but also large differences in mass distribution (archives on upper stories). MDR's for irregular buildings can be several times as high as for regular ones. Structures with soft stories, e.g. without any wall in the ground level floor, have proved to be very vulnerable. Frequently the columns of this level collapse, leading to a total loss of the building.

For our example, we will use the MDR's (in $\%$ of value) published by F. Sauter (see Table 2):

TABLE 2: EARTHQUAKE RISK ASSESSMENT

\begin{tabular}{|c|c|c|c|c|c|}
\hline \multirow[b]{2}{*}{ Building Type } & \multicolumn{4}{|c|}{ Intensity (MM) } & \multirow[b]{2}{*}{$\mathrm{X}$} \\
\hline & VI & VII & VIII & IX & \\
\hline Adobe & 8 & 17 & 50 & 100 & 100 \\
\hline Unreinforced masonry - low quality & 4.5 & 14 & 40 & 80 & 100 \\
\hline Reinforced concrete frames without seismic design & 2.5 & 12 & 33 & 70 & 100 \\
\hline Steel frames without seismic design & 2 & 6 & 18 & 41 & 72 \\
\hline $\begin{array}{l}\text { Reinforced masonry, medium quality, without } \\
\text { seismic design }\end{array}$ & 1.5 & 5.5 & 16 & 38 & 66 \\
\hline Reinforced concrete frames with seismic design & 1 & 4 & 13 & 33 & 60 \\
\hline Shear walls with seismic design & .6 & 1.5 & 7 & 17 & 30 \\
\hline Wooden frame dwellings & .5 & 3 & 8 & 15 & 23 \\
\hline Steel frames with seismic design & .4 & 2 & 7 & 20 & 40 \\
\hline $\begin{array}{l}\text { Reinforced masonry, high quality, with seismic } \\
\text { design }\end{array}$ & .3 & 1.5 & 5 & 13 & 25 \\
\hline
\end{tabular}




\section{Cover conditions}

The earthquake peril is usually covered with an endorsement to the fire policy. Cover conditions vary from one country to another, as already pointed out in a previous chapter. Because the cover conditions can have a strong impact on the risk assessment, we try to give a more or less complete list of items that are mentioned in the context of earthquake insurance.

With regard to insured perils, we have to mention

- Material damage directly caused by the earthquake shock (cracks in building walls) or as consequence of shaking (settlement, tilting, liquefaction, earthslides).

- Fire following earthquake: San Francisco 1906 , Tokyo 1923.

- Tsunami. High energy wave caused by events rupturing the sea-bottom. Epicenter and place of impact can be several thousand kilometers apart.

- Volcanic eruption. Damage can be caused by lava flows, mud flows (Nevado del Ruiz 1985, Mount St. Helens 1980), ash deposits and corrosive gases (over very large distances as experienced during the Katmai eruption in Alaska 1912).

- All risks cover.

Differences between countries also can be found in the interests insured:

- Buildings, with inclusion or exclusion of foundations, retaining walls, frescos, mosaics etc.

- Contents, stock, equipment;

- Sprinkler leakage;

- Business interruption (LOP);

and especially in the type of cover

- Full value insurance (indemnification of repair costs or replacement value)

- Time (indemnity) value insurance (deduction of depreciation).

- First loss policy; losses indemnified up to agreed amount (usually sublimits of fire sums insured for catastrophe perils).

- Deductible (sometimes franchise, where losses are fully indemnified if they exceed franchise amount).

- Layered policy (with deductible and loss limit).

- Coinsurance (insured participates with agreed percentage on each loss).

Due to the possibility of combining some of the above items, cover conditions found in practice are countless. Let's mention some practical cases and express some thoughts instead of trying to analyse different policy types.

- Political influence. After an earthquake in a state with a monopoly insurer, the flat amount deductible was reduced to $40 \%$ of the figure mentioned in the policy wording. The same has happened in other countries with other perils.... Consequences: Depending on reinsurance program, either insurer and reinsurer (proportional) or the insurer and/or the excess of loss reinsurer (retained business) bear a larger share of the loss and - even as important - a much larger administrative burden and higher loss adjustment expenses.

- Court decisions. After the coalinga earthquake in 1983 an insurance company was condemned to pay earthquake losses under an all risks policy. Earthquake as such was excluded, but not faulty design (concurrent causation).

- Shifting of a claim from one line to another. After bush fires in Australia insurance companies sued the electricity supplier, claiming that the fires were caused due to the negligence to cut back bushes along the power line. Fire claims were converted into liability losses.

- How will losses he handled where the fire policy excludes earthquake shock but not fire following if there is partial collapse with ensuing fire?

- Problematic BI covers. At the moment, roughly half of the sums insured exposed to earthquake in $\mathrm{NZ}$ stem from loss of profit covers. This type of insurance has to be considered as very problematic for the following reasons: BI covers are designed for a situation present after a fire that hits one single risk. It therefore is possible to make all possible means available to put the plant back into operation as fast as possible. This assumption is not valid after an earthquake (or any catastrophe loss) where many risks are affected at the same time and everybody is looking for spare parts and labour force. Government bodies may cordon off areas for security reasons or to reduce or prevent pilferage. Reconstruction or repair may be delayed because of new (emergency) design requirements or prohibition of reconstruction (in zones of poor subsoil) as happened in Mexico after the 1985 event. It is very questionable whether insurance is of much help when reconstruction is finished but the market has been host to competitors.

There are many more reasons which make this cover questionable: Extension to failure of supply or demand, leading to interdependence of different marketplaces like NZ and Japan, very small deductibles (delay period), long insurance periods, very low to nonexisting rates for inclusion of earthquake. Business interruption covers were one of the largest loss producers in the 1976 Guatemala event. Many hotels had to close down due to necessary repair works even without structural damage. In June 1986,36 of the hotels in Mexico City being affected by the september 1985 earthquake were still closed or under repair, only few or none of them carrying BI insurance. But there is no need for building damage to cause huge BI losses. Guatemala is again an example, where a machine was brought out of plumb by the shaking and only could be fixed by specialists that had to be flown in from abroad. Before readjustment began, the BI cover was already working. Is it really the intention to guarantee the turnover 
during the first month after an earthquake? What would happen, if the Edgecumbe earthquake were to occur in the most industrialised part of NZ?

- Problem of two different policies on the same risk. Nobody in the insurance industry was able to explain before the Edgecumbe event, how the EQWDC indemnity cover would work - as a first risk underlying the private reinstatement policy or each policy bearing its share of the material damage in proportion to the corresponding sums insured. Similar problems in cover interpretation and loss adjustment have to be expected in countries, where comparable situations exist.

\section{CALCULATION OF RISK PREMIUMS}

In the last chapter we have prepared the ground for answering one of the questions raised at the very beginning.

In principle, the procedure is straightforward. We just have to know the location of the insured risk and the building type, assuming full value insurance and average subsoil conditions.

For a wooden frame dwelling situated in Wellington we would have to charge a risk rate of

$$
\frac{0.5}{6}+\frac{3}{21}+\frac{8}{67}+\frac{15}{220}+\frac{23}{700}=0.45 \%
$$

(the return period for intensity $\mathrm{X}$ has been assumed to be 700 years). To the risk rate we have to add a loading for uncertainty and fluctuation, the cost and a margin for profit. The risk rate for the same building type in Auckland amounts to $0.03 \%$ and in Christchurch to $0.27 \%$ (assuming a return period of 1800 years for intensity $X)$. For a low quality unreinforced masonry building we would have to charge a risk rate of $2.5 \%$ if the building is situated in Wellington, quite a substantial figure!

If we introduce a deductible of $2 \%$ of the sum insured (full value), and if we assume a lognormal loss distribution, the risk rate for the wooden dwelling in Wellington would reduce to $0.37 \%$ which corresponds to a deductible credit of about $18 \%$.

A relationship similar to the one we used to calculate building risk rates is not available, neither for contents nor for Business Interruption. For normal contents like furniture etc, first investigations lead to the conclusion that MDR's are about half those of a medium quality reinforced masonry building. Vulnerability of contents not only depends on their own damage characteristics; swaying of the building, production of dust, falling of suspended ceilings, collapse of partition walls etc also are decisive for the damage potential. The situation is even less transparent for Business Interruption and has already been discussed elsewhere.

\section{MAXIMUM PROBABLE/POSSIBLE LOSS}

How much is it going to cost? This question is crucial in the context of the ability of the company to continue operating after an event. But it needs further qualification. The above question has to be completed with the addition: and what is the corresponding probability?

Return periods for the different intensities and for a chosen location are the tool we need to answer the above question. For Wellington and reinforced concrete frames with seismic design we will demonstrate the concept.

Intensity Return Frequency Added MDR
Period

$\begin{array}{rrrrr}X & 700 & 0.0014 & 0.0014 & 60 \\ \text { IX } & 220 & 0.0045 & 0.0060 & 33 \\ \text { VIII } & 67 & 0.0149 & 0.0209 & 15 \\ \text { VII } & 21 & 0.0476 & 0.0685 & 4 \\ \text { VI } & 6 & 0.1666 & 0.2352 & 1\end{array}$

If the whole portfolio is located in Wellington and consists of reinforced concrete frames with seismic design, we can interpret the last two columns as follows: A loss of $1 \%$ (or higher) on our exposed values is expected with a yearly frequency of .235; a loss of $60 \%$ of all values exposed is expected with a yearly frequency of .0014. The company has to decide with which frequency they want to work or which EML they want to fix. A very conservative company will choose $60 \%$ and therefore will write only one fourth of sums insured as compared to the company working with a $15 \%$ EML.

The calculation becomes more cumbersome, if we consider not only concrete frames, but also other building types as well as contents and Business Interruption. Further complications arise if our portfolio is not concentrated in one location, but distributed over the whole country. Instead of using return periods, we now have to know how the intensities of an earthquake are spread over the area and in relation to the exposed values. Small earthquakes will not cause problems if the epicenter is located far away from the insured risks, but losses have to be expected as soon as intensity VI is exceeded in one location where part of our portfolio is located. With increasing earthquake magnitude, the area covered with damage producing intensities increases and so does the highest intensity observed. On the other hand, the frequency of very large magnitudes is much smaller. as a result we get a series of potential losses, starting with the MDR for intensity VI applied to the smallest amount of sums insured in our portfolio, and ending with an amount resulting from the "worst case scenario", e.g. largest magnitude with epicenter in the location with the largest portion of the portfolio. As in our introductory example, the company has to choose on which assumed loss potential it wants to underwrite. It is not a scientific but an entrepreneurial decision! 


\section{INFORMATION NEEDS}

We have discussed the ingredients we need to make an earthquake risk assessment. Under this title, we would like to consider items strongly related to insurance and/or reinsurance. We have already mentioned cover conditions, exclusions and the like. In the last paragraph, we have assumed that all information is at hand: sums insured by location and insured interest. In many countries, including $\mathrm{NZ}$, a standardized reporting has been implemented. This reporting is known as CRESTA (Catastrophe Risk Evaluating and standardizing Target Accumulations). A country is divided in earthquake accumulation assessment zones, and for each zone sums insured have to be reported for all insured interests (Building, Contents, Loss of Profit).

The reinsurer needs this information for two reasons. First, to know the geographical distribution of sums insured from all treaties he participates. Second, to set cession limits on target zones, where capacity limits so dictate. With the figures the reinsurer gets from all companies, EML/MPL considerations can be made.

We have looked at earthquake risk assessment from a rather technical point. There are many important problems that need to be considered in setting an underwriting policy. For instance, knowing the amount of money the risk carrier is willing to lose in one event (the capacity), he has to break this figure down for proportional and non-proportional treaty and facultative business. He should think of possible interline accumulations (fire, engineering, workers comp., life...) and reconsider the capacity figure under this aspect. He should make sure that the money he has set away to pay losses is not affected by the event itself and that it can be made available in time (liquidity instead of liquidation!). It still is a problem to build up funds for catastrophe losses that are exempt from taxation.

Last but not least we would like to emphasize the importance of event loss analysis, it's the only real test that our assumptions have to undergo! 